Psoriasis

\title{
Die Versorgung verbessern
}

Eine österreichische Patientenumfrage, die zwischen August 2014 und März 2015 unter der Schirmherrschaft der Österreichischen Gesellschaft für Dermatologie und Venerologie (ÖGDV) durchgeführt wurde, offenbart eine große Unzufriedenheit bei Psoriasis-Patienten. Wenig überraschend hat die Erkrankung großen Einfluss auf das Leben der Betroffenen - wie groß dieser Einfluss ist, wurde aber möglicherweise lange unterschätzt oder - in Ermangelung von Therapiemöglichkeiten - nicht ausreichend thematisiert. Jetzt ist es an der Zeit, verstärkt auf die Patienten zuzugehen und sie über neue Behandlungsoptionen zu informieren.

Laut der österreichweiten Umfrage, an der 1184 Patienten teilnahmen, vermeiden fast $40 \%$ der Psoriatiker Freizeitaktivitäten, $38 \%$ leiden unter einem verminderten Selbstwertgefühl. „Nur für etwa $30 \%$ der Patienten war der Therapieerfolg zufriedenstellend. $70 \%$ empfanden ihre Lebensqualität trotz Behandlung als nicht ganz gut", führte Prof. Dr. Peter Wolf vom Universitätsklinikum Graz auf der Jahrestagung der Österreichischen Akademie für Dermatologische Fortbildung (ÖADF) am 28. Mai in Wels weiter aus.

\section{I) $38 \%$ der Psoriasis-Patienten leiden unter einem verminderten Selbstwertgefühl}

Sein Schluss: „Viele Patienten erhalten derzeit noch keine Systemtherapie. Unser Ziel sollte es sein, die Versorgung der Patienten zu verbessern und somit auch ihre Lebensqualität."

Quelle:

Lunchsymposium Eli

Lilly "Zielgerichtete

Psoriasis-Therapie -

IL-17A-Blockade"

auf der ÖADF-Jahres-

tagung, Wels,

28. Mai 2016

\section{Ixekizumab auf einen Blick}

Ixekizumab (Taltz ${ }^{\circledast}$ ) ist zugelassen zur Behandlung erwachsener Patienten mit mittelschwerer bis schwerer Plaque-Psoriasis, die für eine systemische Therapie in Frage kommen.

Ixekizumab ist ein monoklonaler lgG4-Antikörper, der mit hoher Affinität ( $<3 \mathrm{pM})$ und Spezifität an Interleukin-17 A (IL-17A) bindet. Bei gesunden Menschen trägt IL-17A zum Schutz vor pathogenen Pilzen und Bakterien bei.

Nach derzeitigem Wissensstand spielt das Zytokin IL-17A bei der Pathogenese der Psoriasis eine Schlüsselrolle. IL-17A ist ein Entzündungsbotenstoff, der bei Psoriasis in erhöhten Konzentrationen vorliegt. Das bedingt ein beschleunigtes Wachstum der Keratinozyten, was die charakteristische Plaque-Bildung zur Folge hat. Die Neutralisierung von IL-17A durch Ixekizumab hemmt diese Prozesse.

Verabreichung: Taltz $^{\oplus}$ wird als subkutane Injektion verabreicht, entweder mit einem Fertigpen oder mit einer Fertigspritze. Der Pen ist einfach anzuwenden, sofort gebrauchsfertig und enthält den gelösten Wirkstoff als eine Einzeldosis. Das verwendete Pen-Modell hat sich bereits in der Diabetes-Therapie bewährt.

In klinischen Studien fühlten sich über $90 \%$ der Patienten sicher in der Handhabung der Taltz ${ }^{\oplus}$-Applikationshilfen und bestätigten, dass sowohl der Fertigpen als auch die Fertigspritze einfach anzuwenden sind.

Dosierung: Die empfohlene Dosis beträgt $160 \mathrm{mg}$ mittels subkutaner Injektion (zwei $80 \mathrm{mg}$-Injektionen) in Woche 0 , gefolgt von $80 \mathrm{mg}$ (eine Injektion) in den Wochen 2, 4, 6, 8, 10 und 12; die anschließende Erhaltungsdosis beträgt $80 \mathrm{mg}$ (eine Injektion) alle 4 Wochen.

Ab dem zweiten Behandlungsjahr erfordert Taltz ${ }^{\circledR}$ nur 13 Injektionen pro Jahr - das sind die wenigsten Injektionen aller zugelassenen IL-17A-Inhibitoren

\section{PASI-Ansprechen korreliert mit Lebensqualität}

Studien zeigen, dass der Zugewinn an Lebensqualität umso größer ist, je stärker die PASI (Psoriasis Area Severity Index)-Reduktion ausfällt. „Mit der Verfügbarkeit der neuen Generation an Biologika, wie der Klasse der

\section{PASI-Reduktion mit Ixekizumab} In den Zulassungsstudien UNCOVER-2 und -3 erreichten mit Ixekizumab etwa $90 \%$ der Patienten in den ersten 12 Wochen eine PASI 75-Reduktion, etwa $70 \%$ eine nahezu erscheinungsfreie Haut (PASI 90-Reduktion) und bis zu $40 \%$ eine völlig erscheinungsfreie Haut (PASI 100-Reduktion).

Die hohen Ansprechraten hielten auch in der Erhaltungsphase über 60 Wochen an. Darüber hinaus setzte die Wirkung sehr schnell ein: Schon nach einer Woche konnten sichtbare Ergebnisse nachgewiesen werden. Innerhalb der ersten zwei Wochen lag die mittle- re PASI-Reduktion vom Ausgangswert bei über $50 \%$.

Unter Ixekizumab besserte sich auch die Lebensqualität gemessen anhand des DLQI (Dermatology Life Quality Index). Über $60 \%$ der Patienten, die in der Induktionsphase Taltz ${ }^{\circledR}$ erhielten, gaben an, dass ihre Lebensqualität nicht mehr durch die Plaque-Psoriasis beenträchtigt werde. PA-Ansprechraten erzielen und dam mehr Patienten die Lebensqualit verbessern", so Dr. Leo Richter von der Wiener Rudolfstiftung. 